\title{
Simplified Molecular Input Line Entry Specification
}

National Cancer Institute

\section{Source}

National Cancer Institute. Simplified Molecular Input Line Entry Specification. NCI

Thesaurus. Code C54684.

Specification for unambiguously describing the structure of chemical molecules using short ASCII strings. SMILES strings can be imported by most molecular editors for conversion back into two-dimensional drawings or three-dimensional models for the molecules. 